Mannheimer Manuskripte zu Risikotheorie, Portfolio Management und Versicherungswirtschaft

\author{
Nr. 134 \\ Asset Liability Management bei Versicherungen \\ von \\ Peter AlbReCht
}

Mannheim 09/2001 


\section{Asset Liability Management bei Versicherungen}

1. Einleitung

2. Basisfragen eines ALM

2.1. Interdependenzen zwischen Assets und Liabilities

2.2. Dimensionen und Entwicklungsstufen des ALM

2.3. Determinanten des ALM

3. ALM-Instrumentarium: Makroebene

3.1. Steuerungsgrößen

3.2. Analyseoptionen

3.3. Messung von Risiko und Rendite

3.4. Module und Evaluationsstruktur

3.5. Investmentmodelle

4. Schlußbemerkung und Resümee 


\section{Einleitung}

Die aktuelle Situation des deutschen Versicherungsmarktes ist gekennzeichnet durch steigende Unternehmensrisiken auf der einen Seite bei gleichzeitig steigenden Anforderungen an die Profitabilität der Unternehmen auf der anderen. Deregulierung und Substitutionskonkurrenz führen zu einem verschärften Wettbewerb um Marktanteile bei sinkenden Gewinnmargen. Die anhaltende Niedrigzinsphase an den Kapitalmärkten erschwert die Erwirtschaftung ausreichender Renditen aus laufenden Erträgen. Zum Ausgleich des geringen Zinsniveaus wurde verstärkt in Aktien investiert. Die damit einhergehende Erhöhung der Kapitalanlagevolatilität erfordert eine umfassendere Bewertung und Kontrolle der eingegangenen Risiken, als dies in der Vergangenheit notwendig war. Gleichzeitig führt das Vordringen des Shareholder Value-Gedankenguts zur Forderung einer attraktiven Rendite auf das eingesetzte Kapital.

Asset/Liability-Management (ALM) wird in der Versicherungswirtschaft als ein geeignetes Instrumentarium zur Behandlung der mit der geschilderten Situation einhergehenden Problemstellungen derzeit intensiv diskutiert. ALM beinhaltet im Kern die zielgerichtete Koordination der Steuerung der Aktiva und Passiva, der Abstimmung des Kapitalanlageportefeuilles (Assets) mit den durch die Versicherungsprodukte induzierten versicherungstechnischen Verpflichtungen (Liabilities).

Basisziel eines ALM ist zunächst

* das Management der finanziellen Stabilität

durch Kontrolle der eingegangenen Risikopositionen. Darüber hinaus und auf der kontrollierten Risikoposition aufbauend, tritt hinzu

* das Management der Profitabilität,

ggf. in Form eines Abgleichs bzw. einer Optimierung von eingegangenem Risiko und resultierender Rendite (risikoadjustiertes Performancemanagement ${ }^{1}$, RAPM). Die evaluierten und 
gesteuerten Rendite / Risiko-Positionen betreffen dabei die Produkt- bzw. Investmentebene sowie die Ebene des Gesamtunternehmens.

Bei traditionellen ALM-Techniken, wie Matching- und Immunisierungssstrategien², steht dominant der Stabilitätsaspekt im Vordergrund. Zielsetzung ist die möglichst vollständige Eliminierung des eingegangenen Risikos durch spezifische Anlagestrategien. Die hieraus resultierende näherungsweise risikolose (relativ zu den Verpflichtungen) Position muß aber zwangsläufig mit einer entsprechenden Verringerung der Renditechancen einhergehen. Immunisierungsstrategien sind sicherlich dann sinnvoll, wenn das Versicherungsunternehmen, etwa durch entsprechende Produktgestaltung ${ }^{3}$, selbst ein hohes Risiko eingeht. Eine effektive Ausgestaltung des ALM darf aber nicht allein die Dimension der Risikokontrolle (Deckung der Verpflichtungen) berücksichtigen, sondern muß auch die Renditedimension im Auge haben. Das moderne ALM-Verständnis sieht daher ALM als Teilsystem der finanziellen Unternehmenssteuerung ${ }^{4}$, bei dem sowohl die Wahrung der finanziellen Stabilität als auch die Sicherung der finanziellen Performance im Vordergrund stehen.

Lowe / Stanard (1997, S. 340) sehen die Etablierung und Durchführung eines systematischen ALM als zentrales Instrument zur Erzielung von Wettbewerbsvorteilen (competitive advantages). Sie führen dazu aus:

"Since its formation in 1993, a core strategic premise of the Company has been that an increased level of precision in the measurement and management of risk can be translated into a competitive advantage.

* Improved measurement of underlying exposure and modeling of losses allows underwriters to build a superior insurance portfolio, one that is less risky and / or more profitable than that of peers.

* Improved measurement of financial risk allows management to make more efficient use of capital, leading to superior returns on that capital."

ALM wurde ursprünglich in den USA entwickelt, um Zinsrisiken im Zusammenhang mit zinssensitiven Produkten zu bewältigen. Im Zuge der Entwicklung wurden diese Techniken auf Nicht-Zinsrisiken (z.B. Aktienkursrisiken) ausgedehnt. ALM wurde zu einem wichtigen Kontrollinstrument für produktspezifische, aber auch allgemeine unternehmerische Risiken. 
Techniken, die ursprünglich auf Fragen der Anlagestrategie und Produktentwicklung ausgerichtet waren, wurden angepaßt, um auch andere strategische Bereiche (Rückversicherung, Währungsmanagement) berücksichtigen zu können.

Die Einsatzgebiete des ALM umfassen heute:

* die Liquiditätsanalyse 5

* die Produktanalyse 6

Produkt-Pricing, Produktrisiken, Produktprofitabilität, Reservestellung, Produktdesign das Kapitalmanagement

Solvenztests ${ }^{7}$

effektiver Kapitaleinsatz und Kapitalallokation ${ }^{8}$

sowie allgemein

- die Evaluation und Optimierung von Unternehmensstrategien in den Bereichen

Underwriting $^{9}$ (Pricing, Reserving ${ }^{10}$, Catastrophes)

Investment $^{11}$ (Asset Allocation)

Rückversicherung ${ }^{12}$

ggf. Währung ${ }^{13}$

ggf. Management ${ }^{14}$ (Prämien-, Dividenden-, Marktanteilspolitik)

im Hinblick auf die Rendite / Risikoposition des Versicherers und unter Berücksichtigung der Interaktionen zwischen Kapitalanlagen, Verpflichtungen / Produkten und Kapital- / Reservegrößen.

\section{Basisfragen eines ALM}

\subsection{Interdependenzen zwischen Assets und Liabilities}

Interdependenzen zwischen Assets und Liabilities bestehen zunächst auf der Produktebene (Mikroebene). Im Bereich der Lebensversicherung impliziert hier insbesondere die Produktgestaltung (z.B. Art und Umfang der gewährten Zinsgarantien ${ }^{15}$, Höhe und Form ${ }^{16}$ der Überschußbeteiligung) entsprechende Anforderungen an das Rendite / Risiko-Profil der zu tätigenden Kapitalanlage. Auf der anderen Seite haben die Rendite / Risikoverhältnisse auf den 
Kapitalmärkten, die einzuhaltenden (externen und internen) Kapitalanlagerestriktionen sowie die Kapitalanlagestrategie des Unternehmens Rückwirkungen auf die Finanzierbarkeit und Profitabilität der Produkte.

Im Bereich der Schadenversicherung generieren die Produkte in unterschiedlichem Ausmaß (Short Tail- bzw. Long Tail-Geschäft) Anlagekapital (bilanziell: Schadenrückstellung), das als Zinsträger zur Kapitalanlage zur Verfügung steht. Wie im Bereich der Lebensversicherung haben darüber hinaus die Verhältnisse auf den Kapitalmärkten und die Investmentstrategie Rückwirkungen auf die Profitabilität der Produkte. Hinzu treten weitere Interdependenzen, beispielsweise durch Preispolitik (Cash Flow Underwriting) oder gesetzliche Vorschriften (etwa Abzinsungsgebot für Rückstellungen).

Auf der Unternehmensebene (Makroebene) beeinflussen Korrelationen bzw. Interdependen$\operatorname{zen}^{17}$

* zwischen den Anlageklassen

* zwischen den Versicherungssparten

* zwischen Anlageklassen und Versicherungssparten

die Rendite / Risiko-Position des Gesamtunternehmens.

Generell hängt der finanzielle Status des Versicherungsunternehmens ab von der Höhe der eingegangenen (bzw. in Aussicht gestellten ${ }^{18}$ ) Verpflichtungen einerseits und den Werten sowie dem Renditepotential der den Verpflichtungen gegenüberstehenden Kapitalanlagen. Änderungen der Werte der Investments bedrohen im Extremfall die Deckung der Verpflichtungen und damit die finanzielle Stabilität des Unternehmens, auf jeden Fall beeinträchtigen sie die Profitabilität des Unternehmens. Entsprechend muß das Versicherungsunternehmen die bestehenden Koppelungen zwischen Aktiv- und Passivseite auf der Mikro- sowie der Makroebene im Rahmen seiner finanziellen Steuerung sinnvollerweise berücksichtigen.

Die dargestellten Basiszusammenhänge gelten allerdings nicht erst seit heute, ohne daß das ALM in Deutschland in der Vergangenheit eine besondere Bedeutung entfaltet hätte. Es mußten weitere Faktoren hinzutreten wie etwa ${ }^{19}$ die Deregulierung der Märkte (erhöhter Druck auf die Margen, erhöhter Konkurrenzdruck) sowie die Änderung der Verhältnisse auf den Kapitalmärkten, um das ALM in den Mittelpunkt eines neuen und verstärkten Interesses ${ }^{20} \mathrm{zu}$ rücken. Hinzu kommt sicherlich, wohl aber ausgelöst durch die dargestellten Entwicklungen, 
eine zunehmende Verfügbarkeit von ALM-Tools ${ }^{21}$, die auf die spezifischen Bedingungen des deutschen Marktes angepaßt sind.

\subsection{Dimensionen und Entwicklungsstufen des ALM}

Hinsichtlich unterschiedlicher Dimensionen des ALM können wir zunächst unterscheiden zwischen einem

\section{ALM auf der Mikroebene}

und einem

* ALM auf der Makroebene.

Ein ALM auf der Makroebene geht aus von der Gesamtheit der versicherungstechnischen Verpflichtungen des Unternehmens einerseits (Liability Portfolio) und der Gesamtheit der Kapitalanlagen des Unternehmens (Asset Portfolio) andererseits. Bei einem ALM auf der Mikro-Ebene werden die einzelnen Segmente des versicherungstechnischen Portefeuilles, in der Regel einzelne Produktkategorien oder Produktarten, getrennt betrachtet. Entsprechend wird das durch dieses Segment generierte Anlagekapital separiert ${ }^{22}$. Ein ALM auf der MikroEbene führt daher zunächst zu einer Segmentierung von versicherungstechnischen Verpflichtungen und den zugehörigen Kapitalanlagen. Es erfolgt dann eine spezifische Anlagessteuerung pro Segment zur spezifischen Finanzierung der entsprechenden Verpflichtungen und der Erwirtschaftung von darüber hinaus gehenden Überschüssen.

Als Entwicklungsstufen des ALM können genannt werden

* Traditionelles ALM

* Simultanes ALM

* Total Integrated Risk Management (TIRM) / Integrated Dynamic Financial Analysis

- Value Based Management (VBM) / Risikoadjustiertes Performancemanagement (RAPM) / Holistisches Risikomanagement (HRM)

Das traditionelle ALM, ausgehend von den bereits genannten Matching- und Immunisierungsstrategien, basiert typischerweise auf einer fixierten Verpflichtungsseite und zielt ab auf eine entsprechende Steuerung der Aktivseite. Die Aktiv-Passiv-Steuerung erfolgt dabei rein auf 
der Aktivseite gegeben das Datum der Passivseite und umfaßt die Abstimmung der Kapitalanlagesteuerung auf die Erfordernisse, die von den versicherungstechnischen Verpflichtungen ausgehen.

Ein ALM auf der Makro-Ebene bei fixierten Verpflichtungen entspricht der Thematik der Strategischen Asset Allocation, d.h. der Entscheidung über die anteilige Investition in die Haupt-Anlageklasse (Aktien, Renten, Schuldscheindarlehen, Immobilien, etc.), hier aber unter Berücksichtigung der versicherungstechnischen Verpflichtungen. Angestrebt wird eine Allokation der Anlagemittel auf die Anlageklassen mit dem Ziel, die sich aus den Verpflichtungen ergebenden Anforderungen an die Kapitalanlage zu erfüllen. Zu diesen Anforderungen gehört als Minimalziel die langfristige Sicherstellung der Finanzierung der Verpflichtungen.

Das simultane Asset/Liability-Management hingegen ist ein Teilsystem einer integrierten Gesamtsteuerung des Versicherungsunternehmens und beinhaltet eine wechselseitige Abstimmung des Kapitalanlageportefeuilles einerseits und des Verpflichtungsportefeuilles andererseits. Das simultane ALM im Sinne einer integrierten Portfeuillesteuerung findet insbesondere im Bereich der Schaden / Unfall - (Erst- und Rück-) Versicherung Anwendung und läuft dort im Kern hinaus auf die Bestimmung eines optimalen simultanen Sparten / Anlagen - Mi$\mathrm{xes}^{23}$.

Durch Einbeziehung weiterer Steuerungsaspekte bzw. Unternehmensbereiche, wie die Reserve- und Kapitalpolitik, die Rückversicherungspolitik und das Währungsmanagement, kommt man $\mathrm{zu}$ einer umfassenden und integrierten Unternehmenssteuerung, dem Total Integrated Risk Management ${ }^{24}$ bzw. zu der Integrated Dynamic Financial Analysis ${ }^{25}$.

Der Ansatz des Value Based Managements ${ }^{26}$ bzw. des Risikoadjustierten Performancemanagements ${ }^{27}$ betrifft vor allem die gewählte Zielfunktion. Dabei wird die Verbindung zum Kapitalmarkt hergestellt und die (risikoadjustierte) Eigenkapitalrendite steht im Vordergrund der Unternehmenssteuerung.

Für Ansätze des holistischen Risikomanagements ${ }^{28}$ sind sowohl eine integrierte Unternehmenssteuerung im Sinne des gesamtheitlichen Erfassung aller für das Unternehmen relevanten Risiken und ihrer Interdependenzen als auch eine Integration des Risikomanagements in die Gesamtunternehmenssteuerung charakteristisch. 
Eine Klasse von ALM-Ansätzen, die aktuell verstärkt in den Mittelpunkt der Aufmerksamkeit rückt, ist die

- Dynamic Financial Analysis (DFA).

Charakteristisch für die DFA ${ }^{29}$ ist der explizite Mehr-Perioden-Ansatz, d.h., die Analyse der Finanzlage des Versicherungsunternehmens im Zeitablauf, insbesondere die zentrale Orientierung an Zahlungsströmen (Cash-Flow-Orientierung) als Basis für weitergehende Evaluationen.

\subsection{Determinanten des ALM}

Über die in Abschnitt 2.2 dargestellten grundsätzlichen Spielarten des ALM hinaus existieren eine Reihe von Determinanten, die für die sich in praxi ergebende Ausgestaltung des ALM wesentlich sind. Als Hauptdeterminanten erweisen sich dabei

* die Spartenabhängigkeit des ALM

* die Produktabhängigkeit des ALM

sowie

* die Länderabhängigkeit des ALM.

Dies ist unmittelbar einsichtig, denn die beim ALM zu berücksichtigenden versicherungstechnischen Verpflichtungen weisen entsprechende Abhängigkeiten auf. Zunächst unterscheiden sich die versicherungstechnischen Verpflichtungen in Abhängigkeit von der betrachteten Sparte erheblich in ihrer Fristigkeit, in ihrem Risikoumfang sowie den risikobeeinflussenden Faktoren. Sinnvollerweise unterscheidet $\operatorname{man}^{30}$

* ALM in der Lebensversicherung ${ }^{31}$

* ALM in der Pensionsversicherung ${ }^{32}$

* ALM in der Schaden- / Unfallversicherung ${ }^{33}$ sowie

* ALM in der Rückversicherung ${ }^{34}$.

Der Bereich zur traditionellen Lebensversicherung ist gekennzeichnet durch eine relativ hohe Stabilität der biometrischen Elemente (Stabilität der Rechnungsgrundlagen, Stabilität der Verteilungen des Schadeneintritts und der Schadenhöhe) auf der einen Seite. Ihre Komplexität auf der anderen Seite gewinnt die Lebensversicherung durch ihre z.T. extreme Langfristigkeit 
(mittlere Restlaufzeiten von über 20 Jahren), durch in das Produkt eingehende starke und umfassende Verzinsungsgarantien ${ }^{35}$ sowie durch in das Produkt eingebaute Optionen ${ }^{36}$.

Burghard (1992) sowie Weigel (1991) analysieren die Anforderungen an die Kapitalanlagepolitik deutscher Lebensversicherungsunternehmen primär vor dem Hintergrund des Standardprodukts Kapitallebensversicherung. Die folgenden (teilweise eng miteinander verbundenen) Bestimmungsfaktoren lassen sich im Rahmen dieser Analysen identifizieren: Periodische Erwirtschaftung des geschäftsplanmäßigen Rechnungszinsfußes; periodische Erwirtschaftung einer wettbewerbsfähigen Rendite der Kapitalanlagen; Mindestzuführung zur Rückstellung für Beitragsrückerstattungen (RfB) gemäß den Vorschriften zur Beitragsrückgewähr, Orientierung der Ausschüttung der Überschußbeteiligung an den Höchstwerten der RfB; möglichst hohe (wettbewerbsfähige) und stetige Entwicklung der Überschußbeteiligung; Beachtung der „Verbandsformel“ als Grundlage für Unternehmensvergleiche (Publizitätsaspekt); Risikovorsorge durch Aufbau stiller Reserven; Erwirtschaftung von Deckungsbeiträgen für die Kosten des Neugeschäfts, der Finanzierung der Solvabilitätsvorschriften und der Bedienung der Aktionäre (Shareholder Value).

Förster (1994, S. 389f) behandelt die zentralen Einflußgrößen auf die Verpflichtungen einer Pensionskasse. Er unterscheidet dabei primäre Determinanten, die durch das Trägerunternehmen der Pensionskasse steuerbar sind, von sekundären Determinanten, die gesamtwirtschaftlicher Natur und durch das Trägerunternehmen nicht beeinflußbar sind.

Zur Prognose von Pensionskassen-Verpflichtungen sind vor allem Aussagen über die Bestandsentwicklung erforderlich. Dies bedingt Annahmen über die zeitliche Entwicklung von Sterbens- und Invalidisierungswahrscheinlichkeiten, Verheiratungs- und Pensionswahrscheinlichkeiten, Fluktuationswahrscheinlichkeiten sowie der Zugangswahrscheinlichkeiten, auf deren Basis die Entwicklung des Aktiven- sowie Rentnerbestandes einer Pensionskasse hochgerechnet werden kann. In den Fluktuations- und Zugangswahrscheinlichkeiten bildet sich dabei die Personalpolitik des Trägerunternehmens ab.

Neben der Bestandsentwicklung bildet das Leistungssystem, der materielle Inhalt der Pensionszusagen, die zweite von Förster postulierte Primärdeterminante. Unter dem Leistungssystem versteht Förster dabei die Gesamtheit aller Regelungen, welche u.a. die Leistungsarten, Leistungsformen, Wartezeiten sowie Rentenanpassungen betreffen. Als Leistungsarten 
einer Pensionskasse kommen in Betracht: Altersrenten, Invalidenrenten, Witwen-/ Witwerrenten, Waisenrenten, Sterbegeld sowie andere Notfalleistungen.

$\mathrm{Zu}$ den Sekundärdeterminanten zählt Förster die gesamtwirtschaftlichen Größen Inflation, Zins, Produktivitätsfortschritt, Arbeitsmarktsituation, wirtschaftliche Lage des Trägerunternehmens sowie die Personal- und Vergütungspolitik des Trägerunternehmens.

Diese Sekundärdeterminanten wirken dabei mittelbar auf die Pensionsverpflichtungen ein. Zur Prognose der Entwicklung von Pensionsverpflichtungen müssen daher über die Primärdeterminanten hinaus auch die Sekundärdeterminanten berücksichtigt werden.

Kaufmann / Gadmer / Klett (2001) weisen auf die folgenden Besonderheiten des Bereichs Schaden / Unfallversicherung hin: Inflationssensitivität der Schadenhöhe; stark volatile Schadenverteilungen, insbesondere Existenz von Großschäden; Existenz von Naturkatastrophen; Preiszyklen; stochastischer Schadenentwicklungsprozess; Abhängigkeit der Schadenquote vom der Dauer der Bestandszugehörigkeit (Aging Phenomenon); makroökonomische Einflüs$s e^{37}$

Die vorstehenden Ausführungen unterstreichen nochmals ausdrücklich die Bedeutung der jeweils betrachteten Sparte für die Ausgestaltung des ALM-Systems.

Darüber hinaus bestehen, und dies ist vor allem für ein ALM auf der Mikroebene von Bedeutung, selbst innerhalb der gleichen Sparte z.T. erhebliche Unterschiede hinsichtlich der Produktgestaltung - und damit der Natur der Versicherungsverpflichtungen - in unterschiedlichen Ländern. So weist etwa das Produkt Lebensversicherung mit Überschußbeteiligung deutliche Unterschiede für den deutschen (s.o.) und den britischen Markt auf. Hinzu kommen Produktkonstruktionen, die länderspezifisch sind. ${ }^{38}$

Neben einer Länderabhängigkeit hinsichtlich der Produktgestaltung bestehen auch Unterschiede hinsichtlich des regulativen Umfeldes, die auf die Ausgestaltung des ALM-Systems wirken. Teilweise sind in einzelnen Ländern für bestimmte Sparten bzw. Produkte sogar ALM-Analysen in rudimentärer Form gesetzlich bzw. aufsichtsbehördlich vorgeschrieben. Hierzu zählen das Mismatching-Testing in Großbritannien ${ }^{39}$ und das Cash-Flow-Testing in den $\mathrm{USA}^{40}$. Auch die Risk Based Capital (RBC)-Vorschriften in den USA können als rudi- 
mentäre und pauschale Anwendung eines ALM-Systems auf die Frage der Solvenzsicherung angesehen werden.

Die genannten Gründe sind zugleich Indikatoren dafür, daß ALM-Systeme und -Analysemethoden nicht in unmodifizierter Form von einem Versicherungsmarkt auf den anderen übertragen werden können. Zu unterschiedlich sind oftmals die lokalen Gegebenheiten.

Im Rahmen der weiteren Ausführungen gehen wir auf das Instrumentarium von ALMAnsätzen ein. Dabei beschränken wir uns ${ }^{41}$ auf den Bereich der Makroebene, der Steuerung des Gesamtunternehmens.

\section{ALM - Instrumentarium: Makroebene}

\subsection{Steuerungsgrößen}

Steuerungsgrößen eines ALM-Modells sind regelmäßig zentrale finanzielle Kenngrößen (Performancekennzahlen) des Versicherungsunternehmens entweder als

\section{* Relativgröße}

typischerweise Renditegrößen ${ }^{42} 43$ oder als

* absolute Größe,

beispielsweise Gewinn- bzw. Erfolgsgrößen oder Vermögensendstände oder Bar- bzw. Endwerte. Bei einer DFA-Modellierung ${ }^{44}$ werden zunächst die resultierenden Zahlungsströme generiert und darauf aufbauend die vorgenannten Größen rechnet.

Neben einer modellmäßigen Ermittlung bzw. Projektion von einzelnen Kenngrößen besteht auch die Möglichkeit der Vornahme einer

\section{Bilanzprojektion.}

Die zu evaluierenden finanziellen Kenngrößen können schließlich unterschiedlichen Wertekategorien angehören. $\mathrm{Zu}$ unterscheiden ist zunächst

die Einnahme einer

* (reinen) Marktwertperspektive

von der Einnahme einer 


\section{Rechnungslegungsperspektive.}

Ein für die praktische Unternehmenssteuerung taugliches ALM-Modell muß in der Lage sein, beide Perspektiven zu berücksichtigen. Liegt der Focus auf dem Shareholder Value, so sind die Assets und ggf. auch die Liabilities ${ }^{45}$ zu Marktwerten anzusetzen. Darüber hinaus jedoch existiert ebenso die Notwendigkeit, die Konsequenzen von Unternehmensstrategien auch im Hinblick auf Größen der internen (Kosten-, Leistungs- und Ergebnisrechnung) sowie der externen Rechnungslegung (Gewinn- und Verlustrechnung, Bilanz, Informationen für die Aufsichtsbehörde) überprüfen zu können ${ }^{46}$. So wirken in der Lebensversicherung z.B. die Entwicklung von stillen Reserven und der Rückstellung für Beitragsrückerstattung (freier Teil) zurück auf die darstellbare Aktienquote.

Hinzu treten ggf. weitere Kenngrößen, die außerhalb der bereits genannten Wertekategorien liegen und steuerungsrelevante Informationen enthalten. Im Bereich der Lebensversicherung sei beispielsweise auf die Rückgewährquote oder Kennzahlen zur Kapitalanlageperformance (Verbandsformel, Nettoverzinsung) hingewiesen, im Bereich der betrieblichen Altersversorgung auf den Deckungsgrad einer Pensionskasse. Neben der Ermittlung von einzelnen finanziellen Kenngrößen tritt schließlich die Notwendigkeit der Bestimmung bestimmter strukturierter Zusammenstellungen von Informationsgrößen, vor allem die Erstellung von

\section{Planbilanzen (Bilanzprojektion).}

\subsection{Analyseoptionen}

Hinsichtlich der Analyseoptionen einer ALM-Untersuchung kann grob unterschieden werden in:

* Scenario-Testing (inkl. Stress-Testing)

* Evaluation von Rendite/Risiko-Profilen

* Optimierung.

Bei einem Scenario-Testing findet eine Projektion der Geschäftsergebnisse unter Annahme bestimmter zukünftiger Szenarien statt. Die einzelnen Szenarien sind dabei deterministischer Natur. Im Rahmen von Kapitalmarktszenarien werden bestimmte Renditeentwicklungen von 
Aktienindizes und / oder bestimmte Entwicklungen des Marktzinses bzw. der Zinsstrukturkurve über einen bestimmten Zeitraum betrachtet. Ein Beispiel für das Scenario-Testing ist das bereits angesprochene Cash Flow-Testing. Hierbei werden ${ }^{47}$ sieben standardisierte Bewegungsmuster des Marktzinses über einen Zeitraum von 10 Jahren betrachtet. Eine Variante dieses Ansatzes hat auch auf dem deutschen Versicherungsmarkt Eingang gefunden ${ }^{48}$ im Rahmen der Zinsszenarien der Deutschen Aktuarvereinigung, insbesondere zur Prüfung des Höchstrechnungszinses für die Deckungsrückstellung.

Der Vorteil eines solchen Scenario-Testing ist die einfache Implementierbarkeit und Kommunizierbarkeit sowie der direkte Zugang zum methodischen Ansatz. Das Scenario-Testing ermöglicht die Bestimmung einer Bandbreite (Sensitivitätsanalyse) der betrachteten Geschäftsergebnisse in der Zukunft. Der realisierbare Planungshorizont kann dabei aber nur kurz- bis mittelfristig sein. Im Hinblick auf eine differenzierte Risikoanalyse ist dieser Ansatz allerdings eher rudimentär, da insbesondere keine Wahrscheinlichkeitsvorstellungen über den Eintritt der einzelnen Szenarien spezifiziert werden müssen und Eingang in die Analyse finden.

Eine Variante des Scenario-Testing ist das Stress-Testing, bei dem nur bestimmte besonders adverse Entwicklungsszenarien angesetzt werden (Worst Case-Analyse). Im Fall von Kapitalmarktsszenarien könnte dies etwa ein Einbruch der Aktienkurse und / oder eine massive Veränderung des Kapitalmarktzinses sein. Der bereits angesprochene britische MismatchingTest ist hierfür ein Beispiel. Das Stress-Testing ist eher kurzfristig angelegt und stellt in praxi eine wesentliche Ergänzung der stochastischen Methoden dar.

Im Falle einer Bestimmung bzw. Evaluation von Rendite/Risiko-Profilen ist der zugrundeliegende Ansatz notwendigerweise stochastisch, d.h. es gehen Wahrscheinlichkeitsverteilungen bzw. stochastische Prozesse für die interessierenden Größen bzw. in einer ersten Stufe für die zugrundeliegenden Determinanten dieser Größen ein. Die Auswertung kann dabei je nach Komplexität der Modellierung auf einem analytischen Wege oder aber auf dem Wege einer Monte Carlo-Simulation erfolgen. Auf dieser Basis kann dann der Vergleich unterschiedlicher Geschäftsstrategien hinsichtlich ihrer Wirkung auf die Risiko/Rendite-Position zentraler finanzieller Kenngrößen erfolgen. Darüber hinausgehend kann man versuchen, im Rahmen eines Optimierungsansatzes die bestmögliche oder aber (Markowitz-) effiziente Unternehmensstrategien im Rahmen der getroffenen Modellbildung zu identifizieren. Auch hierbei kann man auf analytischem oder auf dem Weg einer Monte Carlo-Simulation vorgehen ${ }^{49}$. 
Als Optimierungsverfahren kommen im Rahmen von ALM-Ansätzen u.a. zum Einsatz:

* das Markowitz-Verfahren

* das Safety-First-Verfahren

* die direkte Rendite/Risiko-Optimierung.

Bei einer der Optimierung des Markowitz-Typus erfolgt eine Bestimmung des effizienten Randes $^{50}$ (hier: Asset / Liability Efficent Frontier, ALEF) auf einer Basis der Markowitzschen Portfolio-Theorie. Die Liabilities können dabei als eigenständige Asset-Klasse (mit einem negativen Vorzeichen) modelliert werden ${ }^{51}$ - Gegenstand der Optimierung ist dann der Surplus der Assets über die Liabilities - oder werden als Restriktionen (Sicherung der Verpflichtungen) modelliert, dies führt typischerweise zu einer Asset Allocation unter ShortfallNebenbedingungen ${ }^{52}$.

Bei Anwendung des Saftey First-Prinzips ${ }^{53}$ erfolgt formal die Maximierung des Erwartungswertes der betrachteten finanziellen Größe unter der Beachtung von Shortfall-Nebenbedingungen, die wiederum eine Sicherung der Verpflichtungen gewährleisten.

Bei einer direkten Rendite/Risiko-Optimierung wird schließlich der Trade-off zwischen der Performancekonsequenz und der Risikokonsequenz einer Unternehmensstrategie in expliziter Weise vorgegeben ${ }^{54}$.

\subsection{Messung von Risiko und Rendite}

Die stochastischen Ansätze erfordern zu ihrer Evaluation notwendigerweise eine Konzeptionalisierung eines oder mehrerer Performancemaße sowie eines oder mehrerer Risikomaße. Im traditionellen Markowitz-Ansatz dienen als Performancemaß der Erwartungswert und als Risikomaß die Standardabweichung bzw. Varianz. In Weiterentwicklungen und Varianten dieses Basisfalls können Risikomaße des Shortfall-Typus ${ }^{55}$ betrachtet werden:

Shortfall-bzw. Ruinwahrscheinlichkeit

* Shortfall-Erwartungswert bzw. Expected Policyholder Deficit

* Shortfall-Varianz. 
Weitere Risikoaspekte werden erfaßt durch die Größen

* Verteilungsquantile

* Value-at-Risk bzw. risikoadjustiertes Kapital

* Tail Conditional Expectation bzw. bedingter Value-at-Risk ${ }^{56}$.

Lowe/Stanard (1997) führen eine verallgemeinerte Portfeuille-Steuerung durch, indem sie diverse Teil-Performancemaß $\mathrm{e}^{57}$ und Teil-Risikomaße $\mathrm{e}^{58}$ jeweils $\mathrm{zu}$ einem einzigen Performance- bzw. Risikomaß aggregieren und damit wieder in der Markowitz-Welt sind.

\subsection{Module und Evaluationsstruktur}

Nach der Behandlung der zentralen Steuerungsgrößen und den Möglichkeiten ihrer Evaluation wenden wir uns nun der Frage zu, wie die interessierenden Größen generiert werden. Als strukturelle Komponenten (Module) eines ALM-Modells werden dazu benötigt:

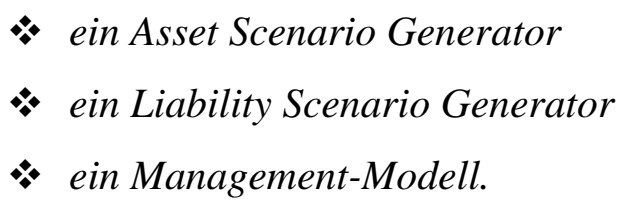

Im Rahmen des Asset Scenario Generators erfolgt eine Projektion der Wertentwicklung des Bestandes an Kapitalanlagen des Versicherungsunternehmens. Hierauf gehen wir vertieft im nächsten Abschnitt ein. Analog erfolgt im Rahmen des Liability Scenario Generators eine Projektion des (typischerweise verdichteten) Bestandes an versicherungstechnischen Verpflichtungen. Verbindungen sind dabei insbesondere vom Asset Scenario Generator zum Liability Scenario Generator möglich. Im Bereich der Lebensversicherung gilt dies etwa für Produkte, bei denen die Höhe der Verpflichtung von Entwicklungen an den Kapitalmärkten abhängig ist (zinssensitive Produkte, aktienindexgebundene Lebensversicherung mit Zinsgarantien) oder für die Abhängigkeit der Überschußbeteiligung von der realisierten Investmentrendite (auf Buchwertbasis). Im Bereich der Schadenversicherung ist daran zu denken, daß die Entwicklung der Inflationsrate sowohl auf die Kapitalmärkte als auch auf die Schadenhöhenkomponente wirkt. 
Ausgewählte ALM-Tools verfügen des weiteren über ein Managementmodul. Dieses enthält eine Zusammenstellung von Entscheidungsregeln ${ }^{59}$, die auf die Assets und/oder die Liabilities wirken. Auf der Assetseite können dies Regeln zur Reinvestionspolitik sein oder zur Realisierung von stillen Reserven, im Bereich der Liabilities die Höhe der zugewiesenen Überschußbeteiligung (deklarierte Überschußbeteiligungssätze). Auf Geschäftsleitungsebene sind Fragen der Bilanzsteuerung, der Dividendenpolitik oder der Preispolitik (z.B. in Abhängigkeit von Marktanteilen, Marktkapazität und ggf. den Preisstrategien der Wettbewerber) betroffen.

Schließlich ist zu unterscheiden, ob und in welcher Weise künftiges

\section{Neugeschäft}

im Rahmen der ALM-Projektion berücksichtigt wird.

Die nachfolgende Abbildung enthält einen strukturellen Überblick über das Ablaufschema einer typischen ALM-Evaluation.

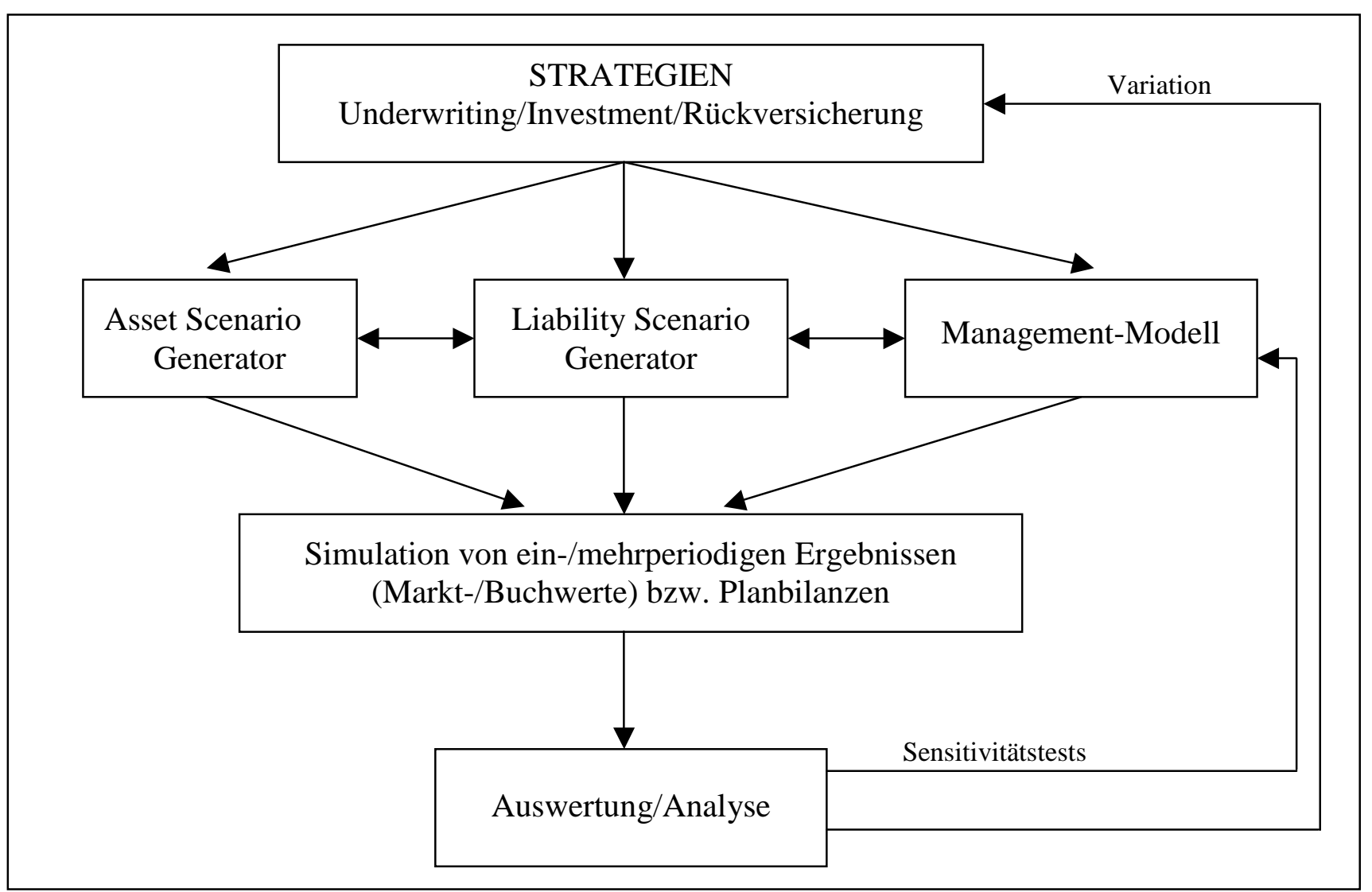

Abbildung: ALM-Evaluation: Allgemeine Struktur 


\subsection{Investmentmodelle}

Eine Projektion der Liabilities gehört zum aktuariellen Standardrepertoire, wohingegen zu einer Projektion der Assets die Expertise weiterer Disziplinen mit eingebunden werden muß. Deshalb soll dieser Punkt im weiteren vertieft behandelt werden.

Die Konstruktion des eingesetzten Investmentmodells wird wesentlich bestimmt von der intendierten Analyseoption (vgl. Abschnitt 3.2). Für ein Scenario-Testing genügt dabei eine Extrapolation von Kapitalmarktszenarien in deterministischer Form, für die Evaluation von Rendite/Risiko-Profilen sind stochastische Investmentmodelle zu konstruieren.

Hierfür bieten Investment- und Kapitalmarkttheorie ${ }^{60}$ sowie Ökonometrie und Zeitreihenanalyse $^{61}$ ein breites Modell- und Methodenarsenal, das auch entsprechend in das ALM Eingang gefunden hat. Stochastische Investmentmodelle für ALM-Zwecke müssen jedoch bestimmten Anforderungen genügen, hierzu gehören etwa die Relevanz ${ }^{62}$ von

\section{* Langfristeigenschaften}

des Modells sowie die Möglichkeit einer

simultanen Modellierung von Assetklassen,

um das Kapitalanlageportfolio eines Versicherungsunternehmens adäquat erfassen zu können. Die Interdependenzen zwischen den Assetklassen können dabei über die Korrelationsstruktur $^{63}$ erfaßt werden oder über eine hierarchische Modellierung ${ }^{64}$ der Modellkomponenten.

$\mathrm{Zu}$ unterscheiden ist ferner, ob das Investmentmodell auf einem

* Total Return Ansatz

basiert oder in der Lage ist, den Total Return in die Komponenten

* laufendes Einkommen bzw. Kursveränderungen

aufzuspalten. Ferner ist von Relevanz, ob das Modell auf

Assetklassen-Ebene (Fondsmodell)

arbeitet oder auf

* Einzeltitelebene. 
Des weiteren muß das Investmentmodell nicht nur Marktwerte, sondern auch Buchwerte generieren können. Eine spezifische Anforderung des deutschen Versicherungsmarktes ist schließlich die Fähigkeit zur Modellierung der volumenmäßig bedeutsamsten Anlageklasse, der Schuldscheindarlehen.

Des weiteren kann als Merkmal die

\section{Dynamikfähigkeit}

des Modells betrachtet werden, d.h. inwieweit ist das Modell etwa in der Lage dynamische (situationsabhängige) Strategien zu berücksichtigen oder auf situationsabhängige Managemententscheidungen innerhalb der Planperiode zu reagieren.

Wir beschließen diesen Abschnitt mit ausgewählten Literaturhinweisen ${ }^{65}$. Von aktuariellen Consultingunternehmen entwickelte Modelle sind das Wilkie-Modell ${ }^{66}$, das CAP: LinkModell $^{67}$, das Russell-Yasuda-Kasai-Modell ${ }^{68}$ und das GRAFT-Modell ${ }^{69}$. Eine Explizierung des zugrundeliegenden Investmentmodells erfolgt des weiteren in den Arbeiten von Stephan ${ }^{69}$ (1995), Yakoubov / Teeger / Duval (1999), Whitten / Thomas (1999), Wengert (2000, S. 132ff.), Pannenberg (2001) und Kaufmann / Gadmer / Klett (2001, S. 222ff.).

\section{Schlußbemerkungen}

Den vorstehenden Ausführungen liegt ein modernes Verständnis von ALM im Sinne eines Teilsystems der finanziellen Unternehmenssteuerung zugrunde. Dabei ist zu beachten, daß ALM „lediglich“ ein Management-Tool darstellt, d.h. die Basis für eine fundierte Entscheidungsfindung liefert, aber nicht zu einer „Automatisierung“ von Entscheidungen führt. Wie bei allen Tools ist ein Verständnis der Prämissen und Methoden erforderlich, um das Tool adäquat einsetzen zu können ${ }^{70}$. Zudem ist das Tool komplex, dies bedingt Verständnis- und Kommunikationsprobleme, aber diese Problematik spiegelt letztlich nur die Komplexität der Aufgabenstellung wider. All dies steht der Einschätzung nicht entgegen, daß sich das ALM zu einem zentralen Tool ${ }^{71}$ für Versicherungsunternehmen zur Kontrolle der finanziellen Stabilität und zur Ausschöpfung finanzieller Potentiale entwickeln wird. 


\section{Endnoten}

${ }^{1}$ Vgl. etwa Albrecht (1998a).

${ }^{2}$ Vgl. in einer Übersicht etwa Albrecht (1998b).

${ }^{3}$ Z.B. Einmalbeitrags-Rentenversicherungsprodukte, bei denen der Garantiezins in der Nähe des Marktzinses liegt oder eine aktiengebundene Lebensversicherung mit Zinsgarantien.

${ }^{4}$ Einer Unternehmenssteuerung auf Basis von finanzwirtschaftlichen Steuerungskriterien.

${ }^{5} \mathrm{Vgl}$. hierzu etwa Wengert (2000, S. 80ff).

${ }^{6}$ Im Bereich der Lebensversicherung gehören hierzu etwa Fragen der Finanzierbarkeit von Rechnungszins, Direktgutschrift und Überschußbeteiligung sowie des Profit Testing.

${ }^{7}$ Zum Komplex ALM und Solvenztests vgl. etwa Hardy (1993) und Berketi / Macdonald (1999).

${ }^{8}$ Zum Komplex ALM und Kapitalallokation vgl. etwa Hodes / Feldblum / Neghaiwi (1999), Cumberworth et al. (2000), Mango / Mulvey (2000) und Philbrick / Painter (2000).

${ }^{9} \mathrm{Vgl}$. hierzu etwa Meyers (2001).

${ }^{10}$ Vgl. hierzu etwa Venter et al. (1998) sowie Kaufmann / Gadmer / Klett (2001, S. 230ff.).

${ }^{11}$ Zum Komplex ALM und Investment-Strategien, insbesondere Asset Allokation, vgl. etwa Stephan (1995), Kaufman / Ryan (2000), Kirschner (2000) Burkett / Mc Intyre / Sonlin (2001).

${ }^{12}$ Zum Komplex ALM und Rückversicherungsstrategien vgl. etwa Venter et al. (1998) und Burkett / Mc Intyre / Sonlin (2001).

${ }^{13} \mathrm{Zu}$ ALM-Anwendungen im Währungsbereich vgl. etwa Sweeney et al. (1998) sowie Blum et al. (2001).

${ }^{14}$ Vgl. etwa Kirschner (2000) und Lowe / Stanard (1997, S. 352ff).

${ }^{15}$ Beispielsweise nur Ablaufgarantien (Riester-Rente) oder Garantien für jedes einzelne Versicherungsjahr (Kapitallebensversicherung).

${ }^{16}$ Etwa möglichst kontinuierliche, schwankungsarme Entwicklung der Überschußbeteiligung (Charakteristik des deutschen Lebensversicherungsmarktes) oder Betonung der Schlußüberschußanteilskomponente (Charakteristik des britischen Versicherungsmarktes).

${ }^{17}$ Eine solche Interdependenz kann z.B. durch die Wirkung eines gemeinsamen Faktors ausgelöst werden. So hat die Inflationsrate einerseits Auswirkungen auf den Kurzfristzins (und damit im Prinzip auf die gesamte Zinsstruktur), auf der anderen Seite besteht in der Schadenversicherung eine Inflationssensitivität der Schadenkosten.

${ }^{18}$ Beispielsweise eine kompetitive Überschußbeteiligung im Bereich der Lebensversicherung.

${ }^{19}$ Weiter zu nennen sind etwa das Shareholder Value - Gedankengut sowie neue Anforderungen an das Risikomanagement durch das KonTraG. Künftig können weitere relevante Einflußfaktoren die Änderung der bilanziellen Rahmenbedingungen (IAS) sowie ggf. Änderungen von Marktusancen durch die „Riester-Rente“ sein.

${ }^{20}$ Zum Stand der Einsatzes von ALM-Tools und -Methoden bei deutschen Versicherungsgesellschaften vgl. aktuell DAV-Arbeitskreis ALM (2001).

${ }^{21}$ Neben Tools von Consulting-Unternehmen und Rückversicherern auch Inhouse-Lösungen

${ }^{22}$ Dies kann im Rahmen einer rein internen Segmentierung erfolgen oder aber explizit durch die Bildung getrennter Anlagestöcke.

${ }^{23}$ Vgl. grundlegend etwa Albrecht (1995, S. 112ff.) und Albrecht / Zimmermann (1992) sowie weiterführend Schradin (1998, Kapitel 3), Maurer (1999) und Maurer / Dus (2001).

${ }^{24}$ Vgl. etwa Correnti / Neulon / Sonlin (1997).

${ }^{25}$ Vgl. etwa Stanard / Lowe (1997).

${ }^{26}$ Im ALM-Kontext vgl. vor allem Corell (1998).

${ }^{27}$ Vgl. etwa Albrecht (1998).

${ }^{28}$ Vgl. etwa Albrecht (1999) und Brohm (2001).

${ }^{29}$ Für den Bereich der Lebensversicherung vgl. zur DFA aktuell Schwarz (2000), für den Bereich der Schadenversicherung Kaufmann / Gadmer / Klett (2001).

${ }^{30}$ Darüber hinaus kann man die Bereiche Krankenversicherung sowie Bausparen betrachten, hierzu gibt es jedoch nur wenige veröffentliche Studien.

${ }^{31} \mathrm{Zu}$ ALM-Anwendungen im Bereich der Lebensversicherung vgl. für die Verhältnisse des deutschen Versicherungsmarktes insbesondere Stephan (1995), Melsheimer (1998), Busson / Ruß / Zwiesler (2000), Förterer (2000), Jaquemod (2000), Junker / Schwarz (2000), Schwarz (2000), Wengert (2000), GE Frankona Re (2000, 2001) sowie Pannenberg (2001). Für aktuelle internationale Beiträge sei hingewiesen auf Consiglio et al (2001 a,b).

32 Zu ALM-Anwendungen im Bereich der Pensionsversicherung vgl. etwa Baum (1996), Brender / van Aalst / Heemskerk (1998), Dert (1998) sowie Nager (1998).

${ }^{33} \mathrm{Zu}$ ALM-Anwendungen im Bereich der Schadenversicherung vgl. man etwa Hodes / Feldblum / Neghaiwi (1999), Maurer (1999), Fromme (2000), Kaufmann / Gadmer / Klett (2001), Maurer / Dus (2001) sowie Schmeiser (2001). 
Starke Impulse zur Entwicklung eines ALM in der Schadenversicherung hat das Projekt des „Public Access DFA-Modell“ der Casualty Actuarial Society entfaltet, vgl. hierzu etwa D’Arcy et al.(1997, 1998) und Walling et al. (1999). Die Proceedings der Foren der CAS sind im World Wide Web verfügbar unter www.casact.org.

${ }^{34} \mathrm{Zu}$ ALM-Anwendungen in der Rückversicherung vgl. etwa Schradin (1998), Bayerische Rück (1999) und Brohm (2001).

${ }^{35}$ Vgl. hierzu etwa Albrecht (2000, S. 1860).

${ }^{36}$ Vgl. hierzu insbesondere Gerdes (1997), Förterer (2000, S. 10ff. , S. 72ff.) sowie Swiss Re (2000, S. 9).

${ }^{37}$ Z.B. Arbeitslosenquote und Kapazitätsauslastung auf Worker's Compensation, Verkehrsdichte und Absatzzahlen im Automarkt auf Kfz-Haftpflichtversicherung.

${ }^{38}$ Vgl. etwa Albrecht (1995, S. 61).

${ }^{39}$ Vgl. etwa Biller (1995).

${ }^{40}$ Vgl. etwa Swiss Re (2000, S. 11ff.).

${ }^{41}$ Zum Instrumentarium eines ALM auf der Mikroebene vgl. etwa Albrecht (1998b, S.101f.).

${ }^{42}$ Investmentrenditen bzw. Kapitalrenditen (Return on Equity, ROE; Return on Risk Adjusted Capital, RORAC).

${ }^{43}$ Eine relative finanzielle Kenngröße, die keine Rendite im üblichen Sinne ist, ist der Deckungsgrad von Assets zu Liabilities (Funding Ratio), vgl. hierzu allgemein etwa Leibowitz / Kogelman / Bader (1994). Der Deckungsgrad spielt insbesondere bei Einrichtungen der betrieblichen Altersversorgung eine wichtige Rolle, vgl. etwa Nager (1998, S. 243 f.)

${ }^{44} \mathrm{Vgl}$. Abschnitt 2.2.

${ }^{45} \mathrm{Zu}$ den Schwierigkeiten einer Marktbewertung der Verpflichtungen im Bereich der Lebensversicherung vgl. GE Frankona Re (2001, S. 25f.).

${ }^{46}$ Auch Kaufmann / Gadmer / Klett (2001, S. 236) weisen darauf hin, daß ein ALM-System unter Umständen mehrere Rechnungslegungskategorien berücksichtigen können muß, z.B. Statutory Accounting und GAAPAccounting.

${ }^{47}$ Vgl. erneut Swiss Re (2000, S. 12f.).

${ }^{48}$ Vgl. Schoch / Eckhardt (1997, S. 187ff.).

${ }^{49}$ Stephan (1995, Kapitel 6 und 7) setzt sogar beide Varianten zur Problemlösung ein.

${ }^{50} \mathrm{Zu}$ einer traditionellen Mittelwert/Varianz-Optimierung vgl. etwa Corell (1998) und Maurer (1999). Variationen hierzu ergeben sich durch die Wahl alternativer Risikomaße, vgl. hierzu etwa Lowe / Standard (1997, S. 345ff.).

${ }^{51}$ Vgl. hierzu allgemein Keel / Müller (1995).

${ }^{52}$ Vgl. hierzu etwa Leibowitz / Bader / Kogelmann (1992).

${ }^{53}$ Im ALM-Kontext wendet etwa Stephan (1995) dieses Entscheidungsprinzip zur Bestimmung der Asset Allocation von Lebensversicherungen an.

${ }^{54}$ Ein Beispiel hierfür bietet das Russel-Yasuda-Kasai-Modell von Carino et al. (1994). Zielfunktion ist hier der Erwartungswert minus einer stückweise linearisierten Shortfall-Strafkostenfunktion. Vgl. hierzu auch Nager (1998, S. 257ff.)

${ }^{55}$ Hierbei wird das Risiko als die Gefahr einer Unterschreitung vorgegebener Targets verstanden, vgl. etwa Maurer (1999, S. 59ff.).

56 Der Tail Conditional Expectation (Tail-VaR, Conditional VaR) mißt die Höhe der mittleren VaRÜberschreitung unter der Bedingung, daß eine solche Überschreitung eingetreten ist. Im Falle stetiger Verteilungen erfüllt diese Größe die Anforderungen von Artzner et al. (1999) an ein kohärentes Risikomaß. Man vgl. zum Tail-VaR des weiteren Embrechts / Resnick / Samorodnitsky (1999, S. 40).

${ }^{57}$ Benutzte Teil-Performancemaße sind z.B. Erfolgsgrößen zu Buch- oder Marktwerten sowie Marktanteile, jeweils absolut und relativ (Kapital, Wettbewerber).

${ }^{58}$ Benutzte Teil-Risikomaße sind: Ruinwahrscheinlichkeit über die nächsten 10 Jahre; Wahrscheinlichkeit einer Schaden/Kosten-Quote über $110 \%$ im nächsten Jahr; Shortfall-Erwartungswert (,Expected Policyholder Deficit“; EPD) des vorhandenen Geschäfts; Wahrscheinlichkeit einer Kapitalverringerung von $20 \%$ oder mehr in den nächsten 3 Jahren; Wahrscheinlichkeit einer Schaden/Kosten-Quote von $2 \%$ oder mehr im Vergleich zum Markt; Wahrscheinlichkeit, daß die Einnahmen um $25 \%$ oder mehr unter Plan sind.

${ }^{59}$ Für den Bereich der Lebensversicherung vgl. zu solchen Entscheidungsregeln etwa GE Frankona Re (2000, S. 9f.), für den Bereich der Schadenversicherung Lowe / Stanard (1997, S. 352ff.).

${ }^{60}$ Z.B. Faktorenmodelle, CAPM / APT, Verbindung von Diffusionsprozessen mit Arbitrage- bzw. Gleichgewichtstheoretischen Überlegungen.

${ }^{61}$ Z.B. ARMA-Modelle oder Vektorautoregressive Modelle.

${ }^{62}$ Etwa im Gegensatz zu den primär kurzfristig orientierten Value at Risk-Modellen.

${ }^{63}$ Dies gilt beispielsweise für das in Corell (1998) sowie das in Wengert (2000) dargestellte Modell.

${ }^{64}$ Dies gilt beispielsweise für das Wilkie-Modell, vgl. etwa Wilkie (1995), oder das CAP: Link-Modell, vgl. etwa Mulvey (1996).

${ }^{65}$ In einem aktuellen Überblick vgl. insbesondere Brohm (2001, S. 225ff.) 
${ }^{66}$ Vgl. etwa Wilkie (1995), die ökonometrische Basis des Wilkie-Modells ist der Box-Jenkins-Ansatz, das Modell verknüpft die Grundgrößen in einer hierarchischen Struktur.

${ }^{67}$ Vgl. etwa Mulvey (1996), Mulvey / Thorlacius (1998); Basis ist hier die Kapitalmarkttheorie, die Grundgrößen werden als Diffusionsprozesse modelliert, hinzu kommt als ökonomische Bedingung der Ausschluß von Arbitragemöglichkeiten. Auch das CAP: Link-Modell besitzt eine hierarchische Struktur.

${ }^{68}$ Vgl. etwa Carino et al. (1994) sowie Nager (1998); das Modell ist ein dynamisches Investmentmodell unter Verwendung von Szenariobäumen und beruht auf der stochastischen dynamischen Optimierung.

${ }^{69}$ Zum GRAFT (Global Return on Assets with Fat Tails)-Modell vgl. etwa Smith (1997); das Modell beruht auf Levy-Prozessen, ist also fähig, sprunghafte Bewegungen zu generieren. Als ökonomische Bedingungen werden Märkte im Preisgleichgewicht betrachtet.

${ }^{69}$ Die ökonometrische Basis des Stephan-Modells sind vektorautoregressive Modelle.

${ }^{70}$ Anforderungen an ein ,,praxistaugliches“ ALM-Modell für versicherungswirtschaftliche Anwendungen erarbeitet Brohm (2001, S. 123ff.).

${ }^{71}$ ALM ist ein zentrales Tool vor allem zur Ausschöpfung der finanziellen Potentiale des Unternehmens, nicht aber das alleinige Tool für Managementaufgaben. Weitere Handlungsoptionen bestehen beispielsweise im Bereich der Prozeßeffizienz, der Vertriebswege, der Kundenbindung oder der Selektion von Geschäftsfeldern.

\section{Literatur}

Ahlgrim, K.C., S.P. D’Arcy, R.W. Gorvett (1999): Parametrizing Interest Rate Models, Casualty Actuarial Society Forum, Summer 1999, Dynamic Financial Analysis, S. 1 - 50.

Albrecht, P. (1995): Ansätze eines finanzwirtschaftlichen Portefeuille-Managements und ihre Bedeutung für die Kapitalanlage- und Risikopolitik von Versicherungsunternehmen, Karlsruhe.

Albrecht, P. (1998a): Was ein Aktuar über Investmentmathematik wissen sollte: Matching und Immunisierung, Der Aktuar 2/98, S. $61-65$.

Albrecht, P. (1998 b): Was ein Aktuar über Investmentmathematik wissen sollte: Asset / Liability-Management (ALM), Der Aktuar 3/98, S. 99 - 103.

Albrecht, P. (1999): Auf dem Weg zu einem holistischen Risikomanagement?, Versicherungswirtschaft 19/1999, S. $1404-1409$.

Albrecht, P. (2000): Die Anlageperformance des Lebensversicherers messen, vergleichen, beurteilen (II), Versicherungswirtschaft 23/2000, S. $1860-1862$.

Albrecht, P., J. Zimmermann (1992): Risikotheoretische Analyse des Versicherungsgeschäfts auf der Grundlage eines stochastischen Gesamtmodells, Transactions of $24^{\text {th }}$ International Congress of Actuaries, Montreal, Vol. 3, S. 27 - 41.

Artzner, P., F. Delbaen, J.-M. Eber, D. Heath (1999): Coherent Measures of Risk, Mathematical Finance 9, S. 203 - 228.

Bayerische Rück (1999): RICASSO: Risiko, Kapitalmanagement und Rückversicherung, München. 
Berketi, A.K., A.S. Macdonald (1999): The effect of the nature of the liabilities on the solvency and maturity payouts of a UK life office fund: A stochastic evaluation, Insurance: Mathematics and Economics 24, S. 117 - 138.

Biller, Th. (1995): Praktische Anwendungen des Asset/Liability Matching bei angloamerikanischen Lebensversicherern, Blätter der Deutschen Gesellschaft für Versicherungsmathematik, Band XXII, Heft 1, S. $71-92$.

Blum, P., M. Dacarogna, P. Embrechts, T. Neghaiwi, N. Niggli (2001): Using DFA for Modelling the Impact of Foreign Exchange Risks on Reinsurance Decisions, Casualty Actuarial Society Forum, Summer 2001, Reinsurance, S. 49 - 93.

Boender, C.G.E., P.C. van Aalst, F. Heemskerk (1998): Modelling and Management of Assets and Liabilities of Pension Plans in the Netherlands, in: Ziemba / Mulvey (1998, Hrsg.), S. $561-580$.

Brohm, A. (2001): Holistische Unternehmensmodelle in der Schaden- und Unfallversicherung, Karlsruhe.

Burghard, P. (1992): Neue Strategien der Kapitalanlage von Lebensversicherungsunternehmen und ihre Auswirkungen, Versicherungswirtschaft, S. 158 - 165.

Burkett, J.C., T.S. Mc Intyre, S.M. Sonlin (2001): DFA Insurance Company Case Study, Part I: Reinsurance and Asset Allocation, Causalty Actuarial Society Forum, Spring 2001, Dynamic Financial Analysis, S. 59 - 98.

Busson, M., J. Ruß, H.J. Zwiesler (2000): Modernes Asset Liability Management, Versicherungswirtschaft, Heft 2/2000, S. $104-109$.

Carino, D.R. / T.Kent / D.H. Myers / C. Stacy / M. Sylvanus / A. Turner / K. Watanabe / W.T. Ziemba (1994): The Russel-Yasuda-Kasai Model. An Asset/Liability Model for a Japanese Insurance Company Using Multistage Stochastic Programming, Interfaces 24, S. $29-49$.

Corell, F. (1998): Value Based Management, Teile I - III, Der Aktuar 1/98, S. 27 - 34, Der Aktuar 2/98, S. 66 - 78 und Der Aktuar 3/98, S. 103 - 114.

Correnti, S., P.A. Nealon, S.M. Sonlin (1997): Total Integrative Risk Management: A Practical Application for Making Strategic Decisions, Joint Day Procedings, 28. Internationales ASTIN Colloquium / 7. Internationales AFIR Colloquium, Cairns / Australien, S. $141-155$.

Cumberworth, M.P., A.N. Hitchcox, W.D. McConnell, A.D. Smith (2000): Corporate Decisions in General Insurance: Beyond the Frontier, British Actuarial Journal 6, S. 259 296.

D’Arcy, S.P., R.W. Gorvett, J.A. Herbers, T.E. Hettinger, S.G. Lehmann, M.J. Miller (1997): Building a Public Access PC-Based DFA Model, Casualty Actuarial Society Forum, Summer 1997, Dynamic Financial Analysis, S. 1 - 40. 
D’Arcy, S.P., R.W. Gorvett, T.E. Hettinger, R.J. Walling (1998): Using the Public Access DFA Model: A Case Study, Casualty Actuarial Society Forum, Summer 1998, Dynamic Financial Analysis, S. 55 - 118.

Consiglio, A., F. Cocco, S.A. Zenios (2001a): The Value of Integrative IRisk Management for Insurance Products with Guarantees, Wharton School, University of Pennsylvania [Download: http://fic.wharton.upenn.edu/fic/wfic/papers/01/0106.pdf].

Consiglio, A., F. Cocco, S.A. Zenios (2001b): Asset and Liability Modeling for Participating Policies with Guarantees, Wharton School, University of Pennsylvania [Download: http://fic.wharton.upenn.edu/fic/wfic/papers/09/0041.pdf].

DAV-Arbeitskreis ALM (2001): Einsatz von Asset Liability Management-Tools und Methoden bei deutschen Versicherungsgesellschaften, Versicherungswirtschaft 7/2001, S. $493-496$.

Dert, C.L. (1998): A Dynamic Model for Asset Liability Management for Defined Benefit Pension Funds, in: Ziemba / Mulvey (1998, Hrsg.), S. 501 - 536.

Embrechts, P., S.I. Resnick, G. Samorodnitsky (1999): Extreme Value Theory as a Risk Management Tool, North American Actuarial Journal 3, S. 30 - 41.

Förster, W. (1994): Die Optimierung der Vermögensrendite einer Pensionskasse, Betriebsberater 6/1994, S. $287-291$.

Förterer, D. (2000): Ertrags- und Risikosteuerung von Lebensversicherern aus finanzmarkttheoretischer Sicht, Karlsruhe.

Fromme, S. (2000): Integriertes Risiko- und Ertragsmanagement für Versicherungsunternehmen: Neue Wege zur strategischen Steuerung eines Versicherungsunternehmens, in: Hehn, E. (Hrsg.): Innovative Kapitalanlagekonzepte, Wiesbaden, S. 105 - 132.

GE Frankona Re (2001): Asset Liability Management: Der finanzwirtschaftliche Ansatz, Der Markt 1/2001, München.

GE Frankona Re (2000): Asset Liability Management, Der Markt 4/2000, München.

Gerdes, W. (1997): Bewertung von Finanzoptionen in Lebensversicherungsprodukten, Der Aktuar 3/97, S. 117 - 124.

Hardy, M.R. (1993): Stochastic simulation in life office solvency assessment, Journal of the Institute of Actuaries 120, S. $131-15$.

Hodes, D.M., S. Feldblum, A.A. Neghaiwi (1999): The Financial Modeling of PropertyCasualty Insurance Companies, North American Actuarial Journal 3, S. 41 - 69.

Jaquemod, R. (2000), ALM-Modell für einen deutschen Lebensversicherer, 20. Tagung Deutsche AFIR-Gruppe, München. [Download: www.aktuar.de/html/images/termine/rjaquemod. pdf] 
Junker, M. G. Schwarz (2000): Simultanes Asset Liability Management: Kompetenz für die Altersvorsorge, Teil I: Versicherungswirtschaft 18/2000, S. 1410 - 1416, Teil II: Versicherungswirtschaft 19/2000, S. $1486-1494$.

Kaufman, A.M., T.A. Ryan (2000): Strategic Asset Allocation for Multi-Line Insurers Using Dynamic Financial Analysis, Casualty Actuarial Society Forum, Summer 2000, Dynamic Financial Analysis, S. 1 - 20.

Kaufmann, R., A. Gadmer, R. Klett (2001): Introduction to Dynamic Financial Analysis, ASTIN Bulletin 31, S. 217 - 249.

Keel, A., H. Müller (1995): Efficient Portfolios in the Asset Liability Context, ASTIN Bulletin 25 , S. $33-48$.

Kirschner, G.S. (2000): A Cost / Benefit Analysis of Alternative Investment Strategies Using Dynamic Financial Analysis Tools, Casualty Actuarial Society Forum. Summer 2000, Dynamic Financial Analysis, S. 22 - 54.

Leibowitz, M.L., L.N. Bader, S. Kogelman (1992): Asset Allocation under Uncertainty, Journal of Fixed Income, September 1992, S. 7 - 19.

Leibowitz, M.L., S. Kogelman, L.N. Bader (1994): Funding Ratio Return, Journal of Portfolio Management, Fall 1994, S. 39 - 47.

Lowe, S.P., J.N. Stanard (1997): An Integrated Dynamic Financial Analysis and Decision Support System for a Property Catastrophe Reinsurer, ASTIN Bulletin 27, S. 339 371.

Mango, D.F., J.M. Mulvey (2000): Capital Adequacy and Allocation Using Dynamic Financial Analysis, Casualty Actuarial Society Forum, Summer 2000, Dynamic Financial Analysis, S. $55-75$.

Maurer, R. (1999): Integrierte Erfolgssteuerung in der Schadenversicherung auf der Basis von Risiko-Wert-Modellen, Karlsruhe.

Maurer, R., I. Dus (2001): Integrated Asset Liability Modelling for Property Casualty Insurance: A Portfolio Theoretical Approach, erscheint in: Rudolf, M., H. Leser (Hrsg.): Asset Liability Management, Wiesbaden.

Melsheimer, F.H. (1998): Die zukünftige Bedeutung des Asset-Liability-Managements für Lebensversicherungsunternehmen, in: Hehn, E. (Hrsg.): Asset Management, Stuttgart, S. $103-116$.

Meyers, G.G. (2001): An Analysis of the Underwriting Risk for DFA Insurance Company, Casualty Actuarial Society Forum, Spring 2001, Dynamic Financial Analysis, S. 195 -220 .

Mulvey, J.M. (1996): Generating Scenarios for the Towers Perrin Investment System, Interfaces $26, S .1-21$. 
Mulvey, J.M., A.E. Thorlacius (1998): The Towers Perrin Global Capital Market Scenario Generation System, in: Ziemba, W.T., J.M. Mulvey (Hrsg.): World Wide Asset and Liability Modelling, Cambridge, S. $286-312$.

Nager, J. (1998): Innovative Ansätze im Asset-Liability-Management, in: Kleeberg, J.M. / H. Rehkugler (Hrsg.): Handbuch Portfoliomanagement, Bad Soden/Ts., S. 239 - 264.

Pannenberg, (2001): ALM und Produktgestaltung, Vortrag, 21. Tagung Deutsche AFIRGruppe, Stuttgart. [Download: www.aktuar.de/html/images/termine-pannenberg 01.pdf]

Philbrick, S.W., R.A. Painter (2001): DFA Insurance Company Case Study, Part II: Capital Adequacy and Capital Allocation, Casualty Actuarial Society Forum, Spring 2001, Dynamic Financial Analysis, S. 99 - 151.

Schmeiser, H. (2001): Risikomanagement von Versicherungsunternehmen nach KontraG, Zeitschrift für die gesamte Versicherungswissenschaft 90, S. $139-159$.

Schoch, H., J. Eckhardt (1997): Rechnungszins für die Deckungsprüfung, Der Aktuar 3/97, S. $184-189$.

Schradin, H.R. (1998): Finanzielle Steuerung der Rückversicherung, Karlsruhe.

Schwarz, C. (2000), Dynamische Finanzanalyse - Werkzeug für das Asset Liability Management, 20. Tagung Deutsche AFIR-Gruppe, München [Download: www.aktuar.de/html/images/termine/cschwarz.pdf]

Smith, A. (1997): Modelling Global Returns on Assets with Fat Tails (GRAFT Model), Proceedings of the Faculty of Actuaries 1997 Investment Conference, Edinburgh, Vol II, S. $517-557$.

Stephan, T.G. (1995): Strategische Asset Allocation in Lebensversicherungsunternehmen, Karlsruhe.

Sweeney, J.C, S.M. Sonlin, S. Correnti, A.P. Williams (1998): Optimal insurance asset allocation in a multi - currency environment, in: Ziemba / Mulvey (1998, Hrsg.), S. 341 368.

Swiss Re (2000): Asset-Liability-Management für Versicherer, Sigma 6/2000, Zürich.

Venter, G.G. (1997): Modelling the Evolution of Interest Rates: The Key to DFA Asset Models, Casualty Actuarial Society Forum, Summer 1997, Dynamic Financial Analysis, S. $135-163$.

Venter, G.G., J.W. Gradwell, M.Q. Ashab, A. Bushal (1998): Implications of Reinsurance and Reserves on Risk of Investment Asset Allocation, Casualty Actuarial Society Forum, Summer 1998, Dynamic Financial Analysis, S. 221 - 272.

Walling, R.J., T.E. Hettinger, C.C. Emma, S. Ackerman (1999): Customizing the Public Access Model Using Publicly Available Data, Casualty Actuarial Society Forum, Summer 1999, Dynamic Financial Analysis, S. 239 - 266. 
Weigel, H.J. (1991): Die Kapitalanlagen der deutschen Lebensversicherer im Spannungsverhältnis zwischen Mathematik, Rendite und Anlagequalität, Festschrift Georg Büchner, Karlsruhe, S. 539 - 549.

Wengert, H.M. (2000): Gesamtunternehmensbezogenes Risikomanagement bei Lebensversicherungsunternehmen, Institut für Finanz- und Aktuarwissenschaften, Ulm.

Whitten, S.P., R.G. Thomas (1999): A Non-Linear Stochastic Asset Model for Actuarial Use, British Actuarial Journal 5, S. 919 - 953.

Wilkie, D.A. (1995): More on a Stochastic Asset Model for Actuarial Use, British Actuarial Journal 1, S. 777 - 964.

Yokoubov,Y., M. Teeger, D.B.Duval (1999): A stochastic investment model for asset and liability management, Joint Day Proceedings, 30. Internationales ASTIN Colloquium 19. Internationales AFIR Colloquium, Tokio, S. 237 - 266.

Ziemba, W.T., J.M. Mulvey (1998, Hrsg.): Worldwide Asset and Liability Modelling, Cambridge. 